\title{
Isolated colon metastasis of vaginal cuff squamous cell carcinoma; a case report
}

\author{
Azar Ahmadzadeh $^{1}$, Shaghayegh Sherafatmand ${ }^{2}$, Mina Mohtadi ${ }^{3}$, Mohammad Momen Gharibvand $^{3 * \mathbb{}}$, Mohammad \\ Bahadoram $^{4}$ \\ ${ }^{1}$ Department of Gynecology and Obstetrics, Ahvaz Jundishapur University of Medical Sciences, Ahvaz, Iran \\ ${ }^{2}$ Department of Surgery, Ahvaz Jundishapur University of Medical Sciences, Ahvaz, Iran \\ ${ }^{3}$ Department of Radiology, Ahvaz Jundishapur University of Medical Sciences, Ahvaz, Iran \\ ${ }^{4}$ Thalassemia and Hemoglobinopathy Research Center, Research Institute of Health, Ahvaz Jundishapur University of Medical Sciences, \\ Ahvaz, Iran
}

\section{*Correspondence to \\ Mohammad Momen \\ Gharibvand \\ Email: momengharibvand-m@ \\ ajums.ac.ir, mohamad. \\ momen2017@gmail.com \\ Received 13 June 2019 \\ Accepted 30 Aug. 2019 \\ Published online 16 Oct. 2019 \\ Keywords: Vaginal squamous cell carcinoma, Colon \\ invasion}

Citation: Ahmadzadeh

A, Sherafatmand S, Mohtadi M, Gharibvand

MM, Bahadoram M Isolated colon metastasis of vaginal cuff squamous cell carcinoma; a case report. Immunopathol Persa. 2021:7(1):e01. DOI:10.34172 ipp.2021.01.

\begin{abstract}
Primary vaginal squamous cell carcinoma (SCC) is one of the rarest kinds of cancer in gynecological cancer. We report a 40-year-old presented with abdominal pain in her right lower quadrant with past-medical history of vaginal cuff SCC without any metastasis about 2.5 years ago which was treated with a combination of surgery and radiotherapy. Ultrasonography and abdomino-pelvic CT scan suggested an inflammatory phlegmon in RLQ probably due to a complicated appendicitis or malignancy. Ultrasonography-guided biopsy was performed which showed a poor differentiated SCC. Chest and abdominopelvic CTs showed no metastasis. Patients underwent laparotomy and ileocecal resection then end-to-end anastomosis was performed. A study of specimens showed a non-keratinizing SCC.
\end{abstract}

\section{Introduction}

Primary vaginal carcinoma is one of uncommon cancers in gynecological field which represented about $1-3 \%$ of all these malignancies. Studies showed that the incidence of vaginal carcinoma is about $0.42-$ 0.6 per 100000 women in the United States (1, 2 ) and most of the patients are about 60 years old at the time of diagnosis. There are different types of carcinoma in which squamous cell carcinoma (SCC) is the most prevalent vaginal carcinoma. The most common risk factor for vaginal SCC is human papillomavirus (HPV) type 16 and 18 infections (3). To the best of our knowledge, there is no case description of vaginal SCC with isolated ileocecal metastasis. The aim of this study is to present a rare case of ileocecal metastasis of vaginal cuff SCC.

\section{Case Presentation}

A 40-year-old woman gravida 2, para 2 was referred to our clinic with the main complaint of right lower quadrant (RLQ) pain lasting for about three months without any change in bowel habits. She had a past-medical history of cervical cancer which her medical documents revealed. She had undergone CIN III and hysterectomy approximately 44

\section{Key point}

In each patient with history of cancer presenting with new mass in other site, metastasis should be considered.

months ago, and 15 months later she was referred with a vaginal cuff mass which was vaginal cuff high grade non-keratinizing SCC without any distant metastasis. She underwent a subtotal vaginectomy, lymph node dissection, and bilateral salpingooophorectomy. All pathological studies on biopsy's specimens such as lymph nodes, the margin of the resection sites, and omentum were free. Then, the patient received 25 sessions of radiotherapy and five sessions of weekly cisplatin $\left(40 \mathrm{mg} / \mathrm{m}^{2}\right)$. During the postoperation follow ups, every three months, pap smears and physical examinations were conducted. All of the follow-up evaluations were normal. She did not have any co-morbidities, while family history of malignancy was negative too. She did not have any fever or rectal bleeding, however a $4 \mathrm{~kg}$ weight loss over the past three months was noticed. Over the past three months, she

Copyright (C) 2021 The Author(s); Published by Nickan Research Institute. This is an open-access article distributed under the terms of the Creative Commons Attribution License (http://creativecommons.org/licenses/by/4.0), which permits unrestricted use, distribution, and reproduction in any medium, provided the original work is properly cited. 
only got analgesic therapy by local physicians without any further evaluation. Since her condition had deteriorated, the patient was referred to our medical center. Physical examination on admission showed mild tenderness with a mass of hard consistency, non-mobilized in the RLQ with normal vital signs. No other abnormalities were found in physical examination. Ultrasonography of the abdomen and pelvis showed heterogeneous hypoechoic mass, measuring $100 \times 92 \times 73 \mathrm{~mm}$ with inflammatory changes near small bowel loops suggestive of phlegmon presumably due to complicated appendicitis or malignancy. Abdominopelvic CT scan also showed a round ill-marginated opacity with peripheral enhancement in delay phase with small gas bubbles and small eccentric cystic component suggesting an inflammatory process probably due to Crohn's disease, tuberculosis, or malignancy (Figure 1). An ultrasoundguided biopsy was conducted. Histopathology evaluation on specimen confirmed a poor differentiated SCC (Figure 2). Positron emission tomography/computed tomography (PET/CT) was performed to make sure that other sites were free. Finally, she underwent laparotomy which showed a terminal ileum tumor with the approximate size of $100 \times 75 \mathrm{~mm}$ with the mesocecum extension. Ileocecal resection and end-to-end anastomosis were performed. Additionally, some peritoneal and parietal biopsy was performed. All other organs such as small intestine, bladder and omentum were normal during the surgery. Microscopic evaluation of the biopsies revealed high grade SCC in mesenterium involving ileum wall extending to mucosa, however all resected lymph nodes and appendix were free. The patient was discharged from the hospital 10 days after surgery.

\section{Discussion}

Carcinoma of the vagina is one of the rarest gynecological cancers which is mostly due to a primary origin of elsewhere, since the primary carcinoma of the vagina is rarer. Vagina is a common site of metastasis of primary cancer of cervix, ovary, endometrium and gestational trophoblast neoplasia. Approximately 90\% of primary vaginal carcinoma consists of SCC. Other types of vaginal carcinoma are melanoma, endodermal sinus tumor, adenocarcinoma, and embryonal rhabdomyosarcoma. The dominant symptom is the postmenopausal vaginal bleeding (4). Other frequent symptoms are post-coital bleeding, vaginal discharge and dyspareunia. For the diagnosis of primary carcinoma of vagina, first of all, we should rule out the cancer of vulvar, cervical, and urethral cancer because the vagina is a common site of these cancer's metastasis. Most of the primary vaginal SCC occurs in post-menopausal females, especially in women aged 65 years old or more during diagnosis. HPV or human immunodeficiency virus (HIV) infection, smoking, DES (diethylstilbestrol) exposure during pregnancy, multiple sexual partners, uterine prolapse and a history of cervical neoplasia are some risk factors for carcinoma of the vagina
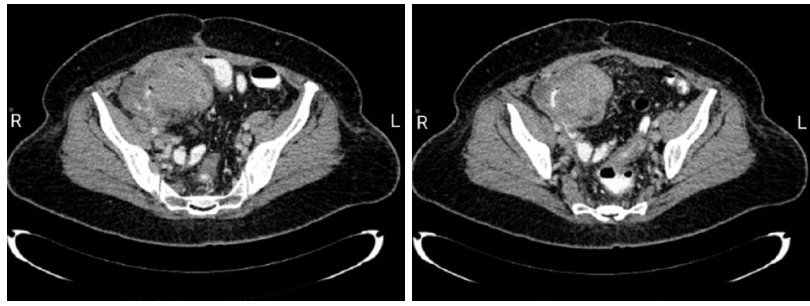

Figure 1. CT scan with both IV and oral contrast shows a hypodense mass with heterogeneous enhancement encasing the bowel loop in right lower quadrant.

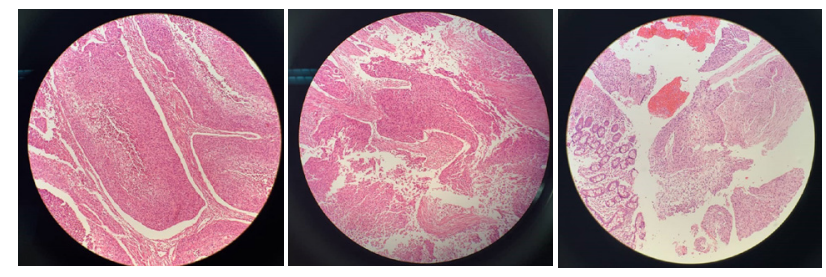

Figure 2. Distal ileum; Deposit of large cell non keratinizing squamous cell carcinoma in mesenterium involving ileal wall extending to mucosa, appendix vermicular is free. All 6 resected lymph node are free.

$(5,6)$. Different studies suggested dissimilar treatment for vaginal carcinoma and individualized treatment suggested, nonetheless most of them suggested surgery, or radiotherapy or a combination of them for stage I and stage II or more with radiotherapy $(7,8)$. Creasman et al evaluated 4885 cases with vaginal cancer in the national cancer registry. They found that the dominant treatment for primary vaginal cancer is radiation except for patients with carcinoma in situ where surgery may be a proper treatment (9).

Different studies also showed that the five-year survival rates of vaginal SCC are about $85 \%, 75 \%$ and $57 \%$ for stage I, stage II, and stage III/IV, respectively $(10,11)$. We reported a case of a 40 - year-old female with a past medical history of vaginal cuff SCC who had been treated by surgery about 2.5 years ago with completely free of margin and other site's metastasis, presented with RLQ pain which after work-ups, a poorly differentiated SCC in ileocecal region have been detected. Our patient was younger than the peak age of vaginal SCC which in Dominico et al showed that only $10 \%$ of vaginal cancers occur in patients with the age of fewer than 40 years old. Some studies showed less than 10 patients with the age younger than 20 years old $(12,13)$. Dominico et al explained that vaginal cancers in younger patients may occur due to their partner's frequency or numbers (13). Literature reviews revealed that about $30 \%$ of patients with vaginal cancer had a history of past cervical cancer $(14,15)$, which is similar to our patient's medical history, where there is a past-medical history of SCC of cervix before vaginal cuff cancer. Our patients after completion of cure were presented with an uncommon RLQ mass which was more suggestive of phlegmon due to complicated 
appendicitis. There was not any invasion to lymph nodes or other abdominopelvic organs. Eltabbakh et al reported a patient with an unusual clinical presentation of vaginal cancer which her symptoms, examinations and imaging suggested an ovarian malignancy; but after evaluations, they noticed that it is a primary vaginal SCC (16). Additionally, Dixit et al studied 70 cases of vaginal SCC. In their two-year follow-ups, distant metastasis was seen only in three patients which were in their pelvic, lung, and vertebrae (17). In another study of 29 patients with vaginal SCC after treatment, 10 patients showed local recurrence while only two patients showed distant recurrence (18). Likewise, Hacker et al showed that the most common sites of vaginal cancer are lungs, liver, and bony skeleton (19).

\section{Conclusion}

Although we often expect a patient with a history of vaginal SCC to present with local manifestations such as lymph nodes or bladder invasions, the patient can be presented with an abnormal manifestation, such as an isolated ileocecal metastasis.

\section{Authors' contribution}

AA, SS, MM conceptualized, treated and followed up the patient. MMG and MB conceptualized, did literature survey and wrote the case report. All authors read and approved the final manuscript.

Conflicts of interest

The authors declare that they have no competing interests.

\section{Ethical considerations}

Ethical issues including plagiarism, double publication, and redundancy have been completely observed by the authors. The patients gave her consent to publish as a case report.

\section{Funding/Support}

This study was supported by grants from vice chancellor for research affairs, Ahvaz Jundishapur University of Medical Sciences, Ahvaz, Iran.

\section{References}

1. Henson D, Tarone R. An epidemiologic study of cancer of the cervix, vagina, and vulva based on the Third National Cancer Survey in the United States. Am J Obstet Gynecol. 1977;129:35.

2. Begum N, Ara I, Islam F, Ganguly S, Afroj S. Primary vaginal carcinoma in prolapsed uterus. J Bangladesh Coll Phys Surg. 2012; 30: 181-2.

3. Daling JR, Madeleine MM, Schwartz SM, Shera KA, Carter J, McKnight B, et al. A population- based study of squamous cell vaginal cancer: HPV and cofactors. Gynecol Oncol. 2002;84:263- 70. doi: 10.1006/gyno.2001.6502.

4. Begum S, Akhter R, Begum K. Primary vaginal carcinoma, tales of tragedy: case report. Chattagram Maa-O-Shishu Hospital Medical College Journal. 2013;12:664-6. doi: 10.3329/ cmoshmcj. v12i3.16718.

5. Benedet JL, Bender H, Jones H 3rd, Ngan HY, Pecorelli S. FIGO staging classifications and clinical practice guidelines in the management of gynecologic cancers. FIGO Committee on Gynecologic Oncology. Int J Gynaecol Obstet. 2000;70:20962. doi: 10.1016/S0020-7292(00)90001-8.

6. Karateke A, Tugrul S, Yakut Y, Gurbuz A, Cam C.Management of a case of primary vaginal cancer with irreducible massive uterine prolapse--a case report. Eur J Gynaecol Oncol. 2006;27: 528-30.

7. Tjalma WA, Monaghan JM, de Barros Lopes A, Naik R, Nordin AJ, Weyler JJ.The role of surgery in invasive squamous carcinoma of the vagina. Gynecol Oncol. 2001;81:360-5. doi: 10.1006/gyno.2001.6171.

8. 8.Platta CS, Anderson B, Geye H, Das R, Straub M, Bradley $\mathrm{K}$. Adjuvant and definitive radiation therapy for primary carcinoma of the vagina using brachytherapy and external beam radiation therapy. J Contemp Brachytherapy. 2013;5:7682. doi: 10.5114/jcb.2013.36177.

9. Creasman WT, Phillips JL, Menck HR. The National Cancer Data Base report on cancer of the vagina. Cancer. 1998;83:103340. doi: 10.1002/(SICI)1097-0142(19980901)83:53.0.CO;2-6.

10. Blecharz P, Reinfuss M, Jakubowicz J, Piotr S, Wysocki W, Karolewski K, et al. Prognostic factors in patients with primary invasive vaginal carcinoma. Ginekologia Polska .2012; 83:904-9.

11. Andersen ES. Primary carcinoma of the vagina: a study of 29 cases. Gynecol Oncol. 1989;33: 317-20.

12. Bivens MD. Primary carcinoma of the vagina; a report of forty-six cases. Am J Obstet Gynecol. 1953;65:390-9. doi: 10.1016/0002-9378(53)90443-8.

13. Di Domenico A. Primary vaginal squamous cell carcinoma in the young patient. Gynecol Oncol. 1989; 35:181-7. doi: 10.1016/0090-8258(89)90040-1.

14. Eddy GL, Marks RD Jr, Miller MC, Underwood PB Jr. Primary invasive vaginal carcinoma. Am J Obstet Gynecol. 1991;165:292-8. doi: 10.1016/0002-9378(91)90081-2.

15. Peters III WA, Kumar NB,Morley GW. Carcinoma of the vagina. Factors influencing treatment outcome. Cancer. 1985; 55:892-7. doi: 10.1002/1097-0142(19850215)55:4<892::aidcncr2820550430>3.0.co;2-n.

16. Eltabbakh GH, Field JM, Trask CE, McDay JB, Swift PD. Primary vaginal squamous cell carcinoma presenting as a cystic pelvic mass. Gynecol Oncol. 2000;76:213-7. doi: 10.1006/ gyno.1999.5583.

17. Dixit S, Singhal S, Baboo H. Squamous cell carcinoma of the vagina: a review of 70 cases. Gynecol Oncol. 1993;48: 80-7. doi: 10.1006/gyno.1993.1013.

18. Andersen ES. Primary carcinoma of the vagina: a study of 29 cases. Gynecol Oncol. 1989;33:317-20. doi: 10.1016/00908258(89)90518-0.

19. Hacker NF, Eifel PJ, van der Velden J. Cancer of the vagina.Int J Gynaecol Obstet. 2015;131 Suppl 2:S84-7. doi: 10.1016/j. ijgo.2015.06.003. 